\title{
METODOLOGÍA PARA LA CORRECCIÓN ATMOSFÉRICA DE IMÁGENES ASTER, RAPIDEYE, SPOT 2 Y LANDSAT 8 CON EL MÓDULO FLAASH DEL SOFTWARE ENVI
}

\author{
ATMOSPHERIC CORRECTION METHODOLOGY FOR \\ ASTER, RAPIDEYE, SPOT 2 AND LANDSAT 8 IMAGES \\ WITH ENVI FLAASH MODULE SOFTWARE
}

\author{
Heileen Aguilar Arias $^{1}$ \\ Rodolfo Mora Zamora ${ }^{2}$ \\ Christian Vargas Bolaños ${ }^{3}$ \\ Centro Nacional de Alta Tecnología, Costa Rica
}

1 Licenciada en Ingeniería Forestal, investigadora del Programa de Investigaciones Aerotransportadas y Sensores Remotos, Centro Nacional de Alta Tecnología, San José, Costa Rica. Correos electrónicos: haguilar@cenat.ac.cr/heileenaguilar@gmail.com

2 Bachiller en Computación e Informática, desarrollador y soportista del Programa de Investigaciones Aerotransportadas y Sensores Remotos, Centro Nacional de Alta Tecnología, San José, Costa Rica. Correos electrónicos: rmora@cenat.ac.cr/rjmoraza@gmail.com

3 Bachiller en Ciencias Geográficas con Énfasis en Ordenamiento del Territorio, investigador del Programa de Investigaciones Aerotransportadas y Sensores Remotos, Centro Nacional de Alta Tecnología, San José, Costa Rica. Se encuentra actualmente adscrito a la carrera de Licenciatura en Ciencias Geográficas con Énfasis en Ordenamiento del Territorio. Correos electrónicos: cvargas@cenat.ac.cr/chrisva107@gmail.com 
Heileen Aguilar Arias, Rodolfo Mora Zamora, Christian Vargas Bolaños. Atmospheric correction methodology for Aster, RapidEye, Spot 2 and Landsat 8 images with ENVI FLAASH module software

\title{
RESUMEN
}

La corrección atmosférica es un proceso que se aplica a las imágenes digitales, con el propósito de eliminar el efecto de los aerosoles y la radiancia intrínseca que se introduce en el sensor y se ve reflejado en la imagen, como producto de la interacción del sensor con la atmósfera. Con el proceso de corrección atmosférica se logra mejorar la calidad visual de la imagen; así como, eliminar el componente intrusivo de la atmósfera. Este trabajo describe el proceso de corrección atmosférica con el uso del módulo FLAASH del software ENVI, para datos adquiridos por cuatro distintos sensores satelitales: Aster, RapidEye, Landsat 8 y Spot 2. En el caso de los sensores Aster y Spot 2 se muestra la corrección atmosférica para datos ortorectificados en formato GeoTIFF, sin las componentes de longitud de onda asociadas, por lo que se profundiza en el método de inclusión de estos valores en la imagen. Por otra parte, para RapidEye y Landsat 8 se muestra la corrección atmosférica para datos en formato GeoTIFF ortorectificados con las longitudes de onda asociadas en el metadato.

Palabras clave: corrección radiométrica, corrección atmosférica, sensores satelitales, MODTRAN4, ENVI-FLAASH.

\begin{abstract}
The atmospheric correction process is applied to digital images in order to remove distortions induced by aerosols and intrinsic radiance introduced in a sensor and reflected in the image as a result of the interactions between the sensor and the atmosphere. By the atmospheric correction process, it's possible to improve the overall visual quality of an image and to remove the intrusive component of the atmosphere. This paper describes the atmospheric correction process, using ENVI's FLAASH module software, applied to data collected by four different satellite sensors: Aster, RapidEye, Spot 2 and Landsat 8. For Aster and Spot 2, atmospheric correction is shown for orthorectified GeoTIFF data that does not include the associated wavelength components, therefore the process includes the setting of these values in the image. For RapidEye and Landsat 8 , the process is applied to orthorectified GeoTIFF data that includes the wavelengths components attached to the metadata for each image.
\end{abstract}

Keywords: radiometric correction, atmospheric correction, satellite sensors, MODTRAN4, ENVI FLAASH

\section{Introducción}

La teledetección es una técnica que permite obtener información digital de los objetos situados sobre la superficie terrestre, sin necesidad de tener contacto directo con ellos. Para esto, es preciso que entre los objetos y el sensor exista algún tipo de interacción, tal como, el flujo energético proveniente de la energía emitida por el propio sensor; o bien, la reflexión de la luz solar en la superficie terrestre. De esta forma, es posible adquirir información de las cubiertas u objetos por medio de reflexión, emisión o por emisión-reflexión (Chuvieco, 2008).

La reflexión es la forma más importante de teledetección, debido a que el sol ilumina la superficie terrestre la cual refleja esa energía en función del tipo de cobertura presente sobre ella; ese flujo de energía reflejado 
Heileen Aguilar Arias, Rodolfo Mora Zamora, Christian Vargas Bolaños. Metodología para la corrección atmosférica de imágenes Aster, RapidEye, Spot 2 y Landsat 8 con el módulo FLAASH del software ENVI

es recogido por el sensor, que lo transmite posteriormente a un almacenamiento digital. Entre la superficie y el sensor se interpone la atmósfera, que dispersa y absorbe parte de la señal original.

De igual forma, la observación remota puede basarse en la energía emitida por las propias superficies, o bien se podría enviar desde un sensor que fuera capaz, de generar su propio flujo energético. En cualquiera de estos casos, el flujo energético entre la cubierta terrestre y el sensor constituye una forma de radiación electromagnética (Chuvieco, 2008).

Las señales de radiación electromagnética colectadas por los sensores de los satélites en el espectro solar, son modificadas por absorción, dispersión y emisión producidas por gases y aerosoles que viajan por medio de la atmósfera, desde la superficie de la Tierra hasta el sensor (López, 1991).

En el primer caso, la absorción por las moléculas de la atmósfera es un proceso selectivo que convierte la radiación incidente en calor (Rejas, 2008).

En el segundo caso, la dispersión, es el mecanismo dominante y se presenta bajo dos formas distintas: la dispersión de Rayleigh, producida por las moléculas de los mismos gases y la dispersión de Mie o dispersión por los aerosoles, que se produce sobre partículas más grandes (López, 1991; Rejas, 2008).

Por último, la emisión, referente a la radiación propia que emite cualquier cuerpo con temperatura mayor a $-273 \mathrm{~K}$, tiene mayor importancia de estudio en el infrarrojo térmico de los sensores remotos.

Los efectos de estos tres factores se manifiestan en un difuminado de la imagen, disminución de contraste (efecto Rayleigh por bruma) (Tagestad, 2000) y la cantidad total de radiación que llega al sensor. La corrección atmosférica busca entonces disminuir o eliminar estas distorsiones que se introducen en las imágenes producto de la interacción de la atmósfera con el sensor.

Los procedimientos habituales de corrección atmosférica se pueden agrupar en modelos físicos de transferencia radiativa a partir de información obtenida de la propia imagen y métodos de ajuste empírico. En el caso de los modelos físicos de transferencia radiativa, se basan en una simulación de las condiciones de la atmósfera en función de las características físico-químicas de la misma, y el día y la hora de adquisición de la imagen. Si no se dispone de los parámetros físico-químicos, pueden utilizarse modelos estándar (Hedley, 2013). 
Uno de los modelos más conocidos es MODTRAN (MODerate resolution atmospheric TRANsmission), el cual busca eliminar los efectos de la absorción y dispersión, causados por las moléculas y partículas atmosféricas en suspensión, de la radiancia recibida por el sensor y convertir esa radiancia a valores de reflectividad de superficie (Berk, et al. (2000).

FLAASH (Fast Line-of-sight Atmospheric Analysis of Spectral Hypercubes) es un módulo avanzado de corrección atmosférica diponible en el software ENVI (ENvironment for Visualizing Images), el cual se basa en el algoritmo de transferencia de radiación MODTRAN4 desarrollado por Spectral Sciences Inc. (Kruse, 2004). Este módulo se basa inicialmente en la ecuación estándar de radiancia espectral para cada píxel del sensor $(L)$, que se aplica al rango de longitud de onda solar (emisión termal es omitida) y superficies Lambertianas, planas o sus equivalentes (Castillo, 2012).

Según López (1991), es necesario aplicar el proceso de corrección atmosférica cuando se realizan cálculos; o bien, correlaciones donde intervienen diferentes bandas de una imagen multiespectral, ya que la dispersión aumenta inversamente con la longitud de onda y los diferentes canales se verán afectados de un modo diferente; asimismo, cuando se utilizan modelos físicos para relacionar la radiancia en el sensor con alguna propiedad de la superficie, debe entonces estimarse y descontarse la componente atmosférica presente en la señal del sensor. De igual forma, en estudios multitemporales, cuando se comparan propiedades, magnitudes, entre otros, en fechas diferentes, la atmósfera cambia de una fecha a otra y es cuando se debe corregir los valores de las radiancias registradas por el sensor para cada una de las bandas. Además, al interpretar diferencias entre magnitudes relacionadas con la componente atmosférica, como por ejemplo, el estrés en la vegetación y la concentración de clorofila en los océanos, debe aplicarse la corrección del efecto atmosférico.

En el estudio realizado sobre Corrección atmosférica de imágenes Landsat-5: Aplicación al estudio edafológico en la Comarca La Plana de Requena-Utie, por Sobrino, García-Collado, Sobrino y Boluda en el año 1997, las zonas donde los factores formadores del suelo como topografía, geología y altitud son diferentes, la atmósfera influye en los valores de reflectividad final obtenida a partir de las imágenes satélite, por lo que es vital realizar corrección atmosférica para eliminar las interferencias en las correlaciones entre las propiedades del suelo y los datos del satélite. 
Heileen Aguilar Arias, Rodolfo Mora Zamora, Christian Vargas Bolaños. Metodología para la corrección atmosférica de imágenes Aster, RapidEye, Spot 2 y Landsat 8 con el módulo FLAASH del software ENVI

Por otra parte, en la investigación "Amazon forests green-up during 2005 drought” llevada a cabo por Saleska, Didan, Huete y Da Rocha (2007), en la que se emplearon imágenes satelitales MODIS Terra 2005, se mostró que los bosques amazónicos de hoja perenne aumentaron su verdor durante una época de sequía, con lo que aseguraron que, a pesar de la amenaza, pueden ser más resistentes a los cambios climáticos de lo que los modelos asumen. Sin embargo, en un estudio similar "Amazon forests did not green-up during the 2005", realizado por Samanta, Ganguly, Hashimoto, Devadiga, Vermonte, Knyazikhin, Nemani y Myneni (2010), se contradice este hallazgo, pues argumentan que estos datos se vieron perjudicados por alteraciones atmosféricas, debido a que durante la época seca se produce un aumento en los aerosoles en la atmósfera y al utilizar las mismas imágenes corregidas atmosféricamente, los resultados no mostraron aumento en el verdor, por el contrario se encontró una afectación de casi el $60 \%$ de la masa boscosa en los bosques amazónicos debido a la sequía.

En el "Estudio de la influencia de las correcciones a imágenes Landsat ETM+ en la obtención de propiedades de cubiertas vegetales" llevado a cabo por Lamolda (2008), se encontró que para imágenes ETM+ la corrección topográfica no afecta los valores de NDVI (Normalized Difference Vegetation Index), mientras que la corrección atmosférica produce un pequeño sesgo, por lo que se hace necesario calcularla a partir de valores de reflectividad exoatmosférica y no desde los valores crudos digitales de la imagen. Para López (1991), la corrección atmosférica puede no ser necesaria en algunas otras aplicaciones como por ejemplo, en cartografía o en algunas clasificaciones en que se trata de establecer sólo diferencias entre clases dentro de una misma imagen.

En el presente trabajo se expone una descripción metodológica para corregir atmosféricamente imágenes de los sensores Aster (AST14OTH) y Spot 2 sin los valores de longitud de onda asociados a la imagen (necesarios para corregir el efecto de la atmósfera con el módulo FLAASH). Así mismo, se muestra la corrección del efecto atmosférico para imágenes de los sensores RapidEye y Landsat 8 con la componente de longitud de onda asociada al metadato. Se pretende que el lector incorpore la metodología como herramienta clave para Sistemas de Información Geográfica (SIG) y sensoramiento remoto a análisis de vegetación, agricultura de precisión y procesos geoambientales, entre otros. 


\section{Corrección atmosférica}

Los sensores satelitales capturan la información de la cobertura de la tierra obtenida a partir de la intensidad de radiación electromagnética, misma que proviene de la luz del sol reflejada sobre la superficie terrestre, sumada a la energía dispersada y reflejada por la atmósfera, conocida como radiancia. Esta información de intensidad es transformada a través de una ecuación lineal (Kruse, 2004) y almacenada en una representación discreta de números digitales (ND) que tienen una escala artificial definida según las características de almacenamiento de cada sensor específico por ejemplo: 8bits, 12 bits y 16 bits.

Dado que la escala de los ND depende completamente del sensor y que la radiancia percibida por el sensor depende de la luz solar al momento de la toma, no se pueden comparar firmas espectrales en ND de imágenes de sensores distintos o inclusive del mismo sensor si fueron capturadas en momentos diferentes, por lo que es necesario convertir los datos de la escala de ND a una escala espectral normalizada (Edwards, 1998).

La corrección atmosférica busca recuperar la radiancia intrínseca del objeto de estudio, obtenida de la señal recibida por el sensor. Para ello se requiere: 1) convertir los ND de cada banda a valores de radiancia $(L)$, 2) la radiancia se transforma a valores de reflectividad en el techo de la atmósfera "Top of Atmosphere" (TOA) y 3) se obtiene mediante FLAASH la conversión a valores de reflectancia (Kruse, 2004).

Los valores en ND son proporcionales a la radiancia capturada originalmente por el sensor, por lo que es posible recuperar la escala original de radiancia aplicando la fórmula de conversión respectiva de cada banda de la imagen, la cual es calculada durante la calibración inicial del sensor y, por lo general, está disponible junto con la información técnica del sensor. Los efectos de dispersión y distorsión atmosféricos se compensan por medio de fórmulas matemáticas, en este caso planteadas por el modelo MODTRAN4 que, tal como se especificó previamente, es el modelo base de la extensión FLAASH.

En términos generales la corrección atmosférica para FLAASH requiere la recodificación de la imagen (transformación BSQ a BIL), transformación a radiancia e identificación de las bandas y parámetros del sensor. Este artículo se centra en mostrar la serie de pasos básicos que se deben aplicar a las imágenes para hacer la corrección. Se destaca para cada uno de los sensores específicos los procesos que se requiere según el formato de los datos. 
Heileen Aguilar Arias, Rodolfo Mora Zamora, Christian Vargas Bolaños. Metodología para la corrección atmosférica de imágenes Aster, RapidEye, Spot 2 y Landsat 8 con el módulo FLAASH del software ENVI

\section{Conversión a radiancia}

Como se indicó anteriormente, los ND de la imagen son proporcionales a la radiancia, pero son multiplicados por un factor de conversión que permite almacenar los datos en formatos más compactos. Los factores de conversión que revierten este proceso son característicos de cada sensor y por lo general son suministrados por el fabricante. Para revertir la compresión sólo es necesario multiplicar los valores de cada banda por el factor de compresión, ya sea un factor único para toda la imagen o un factor específico por cada banda según haya dispuesto el fabricante del sensor, en ciertos casos incluso proporciona una fórmula que debe aplicarse a los datos para revertir esta compresión.

\section{Recodificación de la imagen}

Previo a la aplicación del proceso de corrección atmosférica los datos deben configurarse en un formato requerido por FLAASH, que incluye un ordenamiento especial de los datos y la inclusión de cierta información clave sobre las características del sensor.

Una imagen de dos colores o más está representada por un arreglo ordenado de valores de intensidad codificado de tal manera que, cuando una aplicación computacional la interpreta, puede obtener inmediatamente los valores correspondientes a cada banda de color. Adicionalmente, toda imagen cuenta con un encabezado que indica cuántas líneas y cuántas columnas tiene, de esta manera es fácil identificar la extensión de la imagen y representarla en dos dimensiones.

Existen tres codificaciones típicas de imágenes: la más sencilla y más común es la de bandas secuenciales o BSQ, por sus siglas en inglés, en la cual la imagen tiene todos los valores de la primera banda seguidos de todos los valores de la segunda banda y así consecutivamente hasta la última banda. La segunda codificación presenta los valores de todas las bandas para el primer punto de la imagen, luego los valores de todas las bandas para el segundo punto y así continúa hasta el último punto de la imagen; esta codificación se conoce como intercalado de bandas por pixel o BIP. El tercer método toma la información de la cantidad de filas y columnas y almacena todos los valores de la primera fila de la imagen para la primera banda, seguidos los valores de la primera fila para la segunda banda y así hasta la última banda; este tipo de codificación se llama 
intercalado de banda por línea o BIL. La mayoría de las imágenes se representan en BSQ; sin embargo, la extensión FLAASH de ENVI requiere que las imágenes se representen en BIL.

\section{Identificación de las bandas}

Como se ha mencionado anteriormente, los datos contenidos en la imagen son una representación digital o discreta de la intensidad de radiancia, que es un fenómeno continuo. La información almacenada en cada banda corresponde a la percepción del sensor en un rango de longitudes de onda. FLAASH requiere que el encabezado de la imagen incluya la longitud de onda del punto medio del rango, así como la distancia entre los valores para los cuáles la percepción del sensor alcanza la mitad de la intensidad, es decir los extremos del "ancho completo a la mitad del máximo" o FWHM (Full width at half máximum) por sus siglas en inglés, tal como lo define Weisstein (2014), este dato por lo general está disponible en la información técnica de cada sensor. Para el caso de los sensores RapidEye y Landsat 8 estos parámetros ya vienen incluidos en los metadatos, pero para los sensores Aster y Spot 2 se deben de incluir, mediante el procedimiento que se expone más adelante.

\section{Remoción de dispersión atmosférica}

Según explican Adler-Golden, Berk, Bernstein y Richtsmeier (1998), el modelo de FLAASH se compone de dos factores que consideran la radiancia reflejada desde la superficie de la tierra hasta el sensor y la radiancia que es dispersada en la atmósfera antes de alcanzar el sensor. Los factores que resuelven esta composición dependen de la posición del sensor y del Sol con respecto a la superficie observada (ambos datos conocidos) y del vapor de agua en la atmósfera, que usualmente es desconocido y debe estimarse o aproximarse según las condiciones de la imagen.

El primer factor representa la radiancia que viaja directo desde la superficie hasta el sensor, para convertir estos valores de radiancia en unidades normalizadas de reflectancia. Es necesario indicar al modelo la elevación promedio del suelo, el tipo de sensor, la altitud del sensor con respecto al nivel del mar, la fecha y la hora de la captura.

El segundo factor corresponde a la radiancia reflejada desde la superficie que ha sido dispersada por la atmósfera antes de alcanzar el sensor. 
Heileen Aguilar Arias, Rodolfo Mora Zamora, Christian Vargas Bolaños. Metodología para la corrección atmosférica de imágenes Aster, RapidEye, Spot 2 y Landsat 8 con el módulo FLAASH del software ENVI

Para calcular estos factores el modelo debe estimar la cantidad de vapor de agua presente en la atmósfera. Algunos sensores cuentan con bandas que cubren longitudes de onda específicas a partir de las cuáles se puede calcular la cantidad de vapor partiendo de la imagen; sin embargo, para la mayoría de sensores esta información no está disponible, por lo que FLAASH cuenta con aproximaciones de los valores de vapor de agua según distintas latitudes y épocas de captura de la imagen. En total son seis modelos atmosféricos que aproximan el vapor de agua, de los cuales para Costa Rica el modelo más apto según ITT $(2009$, p.21) es el modelo tropical (en cualquier época del año), el cual estima que la cantidad de vapor de agua desde la superficie hasta la atmósfera en un área de un centímetro cuadrado es de 4.11 gramos (el equivalente a 4.11 centímetros cúbicos de agua por centímetro cuadrado de superficie).

\section{Compensación de las distorsiones por aerosoles}

El vapor de la atmósfera produce dispersión de la luz de forma homogénea. Los aerosoles y partículas en suspensión, por otra parte, producen distorsiones heterogéneas distribuidas aleatoriamente en la imagen. Al igual que con el vapor de agua, existen longitudes de onda que permiten identificar aerosoles y removerlos automáticamente, tal como se detalla en ITT $(2009$, p.23). Sin embargo, para la mayoría de los sensores estas bandas no están disponibles, en ese caso se debe aproximar este valor dependiendo de las fuentes de influencia presentes en la superficie.

ENVI-FLAASH cuenta con cuatro modelos de aerosoles: el rural aplica para sectores que no están bajo influencia fuerte de coberturas industriales o urbanas, asume que existen dos distribuciones de partículas, gruesas y delgadas, mezcladas en la atmósfera. El urbano asume que un $80 \%$ de las partículas son las mismas que considera el modelo rural y el $20 \%$ restante son de partículas similares al hollín. El marítimo se utiliza para los sectores colindantes con áreas costeras o en áreas continentales con gran influencia de vientos marinos, este modelo asume que existen partículas provenientes del mar y otra parte de las partículas pequeñas del modelo rural. Por último, el modelo troposférico aplica para condiciones de gran nitidez y asume que sólo están presentes las partículas delgadas del modelo rural. 
Para completar la extracción de aerosoles, además del modelo de aerosol, es necesario indicar la visibilidad $(V)$ estimada según las condiciones atmosféricas. Esta se expresa en kilómetros en el rango de 0 a 100 donde una atmósfera clara puede ser de 40 a $100 \mathrm{~km}$, si existe niebla moderada visible en la imagen se estima de 20 a $30 \mathrm{~km}$ y si existe niebla densa se establece de $15 \mathrm{~km}$ o menos. Este valor corresponde a una aproximación de la distancia que sería visible por una persona que mira hacia el horizonte dadas las condiciones exactas de la imagen $(V=3,912 / \beta$ donde $\beta$ es la profundidad óptica horizontal por kilómetro) (ITT, 2009, p.24). Una vez completada la remoción de aerosoles la imagen resultante queda representada en unidades de reflectancia y es posible utilizar esta imagen en estudios con múltiples sensores o múltiples capturas en el tiempo.

\section{Corrección atmosférica de imágenes RapidEye}

Las imágenes RapidEye son procedentes de los sensores con el mismo nombre, los cuales operan comercialmente desde el año 2009. Con cinco satélites en órbita ubicados a $630 \mathrm{~km}$ de altitud y un ancho de barrido de $77 \mathrm{~km}$, se capturan imágenes de color con cinco metros de resolución espacial y cinco bandas en el espectro electromagnético: azul (440-510 $\mathrm{nm})$, verde $(520-590 \mathrm{~nm})$, rojo $(630-690 \mathrm{~nm})$, borde rojo $(690-730 \mathrm{~nm}) \mathrm{y}$ el infrarrojo cercano (760-880 nm) (BlackBridge, 2013a). En el mercado existen tres productos con diferente nivel de procesamiento: nivel 1B que es el producto básico para ser procesado por el cliente. El nivel 3A que contiene correcciones radiométricas, geométricas y del sensor; y por último el nivel 3M que corresponde a mosaicos a color con ortorectificación y equilibrados radiométricamente. Para este artículo se muestra el procedimiento de corrección atmosférica para imágenes con nivel 3A.

La corrección atmosférica para datos con nivel 3A se trabaja para las cinco bandas a la misma vez. Se debe abrir la imagen desde la extensión Open External File y luego seleccionar el sensor RapidEye. Lo primero es realizar la conversión de niveles digitales a radiancia tal como lo muestra la ecuación 1 (BlackBridge, 2013b, p.14).

\section{Radiancia $(L)=N D^{*}$ Factor de escala radiométr $($ Ecuación 1$)$}


Heileen Aguilar Arias, Rodolfo Mora Zamora, Christian Vargas Bolaños. Metodología para la corrección atmosférica de imágenes Aster, RapidEye, Spot 2 y Landsat 8 con el módulo FLAASH del software ENVI

Para ello, se utiliza la herramienta Band Math de ENVI en la cual se debe multiplicar los ND de cada banda de la imagen por el factor de escala radiométrico (radiometricScaleFactor), para ello ND se sustituye por el valor de la banda uno (B1) y el factor de escala se extrae de los metadatos disponibles para la imagen (Castillo, 2012).

El resultado de este proceso es la radiancia de un píxel determinado en la parte superior de la atmósfera $(T O A)$ en unidades de watts por estereorradián por metro cuadrado $\left(\mathrm{W} /\left(\mathrm{m}^{2 *} \mathrm{sr}^{*} \mu \mathrm{m}\right)\right)$. Además, para que el módulo de ENVI pueda leer la imagen y realizar la corrección, se requiere que se realice la transformación del formato BSQ a BIL (para el caso de ENVI la corrección atmosférica se debe correr en el sistema de 32 bits en el cual esta soportado el módulo de corrección atmosférica). Una vez ejecutados estos dos pasos se procede con los datos específicos para realizar la corrección atmosférica.

En el módulo de FLAASH se procede a abrir la imagen que contiene los dos pasos anteriores, dado que para FLAASH se requiere que las unidades de radiancia sean $\mu \mathrm{W} /\left(\mathrm{cm}^{2 *} \mathrm{~nm} * \mathrm{sr}\right)$ el factor de escala a emplear para este caso es de 10 (EXELIS, 2014). Luego, con ayuda de los metadatos se ingresan los valores de la imagen como tipo de sensor (RapidEye), fecha y hora de captura, coordenadas del punto central de la escena, altitud del sensor, elevación del terreno del punto central, tipo de modelo atmosférico, tipo de modelo de aerosol y visibilidad. Para estas imágenes no se aplican las variables de remoción de aerosoles y de agua. Según la capacidad del equipo es recomendable asignar un tamaño de ventana de $500 \mathrm{MB}$ o $1000 \mathrm{MB}$ y el resto de parámetros se dejan por defecto. El resultado es una imagen con corrección atmosférica cuyos valores corresponden a niveles de reflectancia.

\section{Corrección atmosférica de imágenes Aster 14OTH}

Las imágenes Aster en formato AST14OTH tienen 14 bandas ortorectificadas y con corrección radiométrica, escaladas en tonos de 256 niveles digitales ( 8 bits) y 4096 niveles digitales (12 bits) que permiten generar imágenes más compactas. Las bandas V1, V2 y V3N corresponden al espectro visible e infrarrojo cercano o "Visible Near InfraRed" (VNIR) tomadas por un telescopio perpendicular a la superficie de la tierra (nadir) y una banda adicional V3B tomada por un telescopio ligeramente desviado hacia atrás 
que permite generar modelos estereoscópicos. Estas cuatro bandas tienen una resolución de 15 metros de tamaño del pixel y abarcan desde los 520 $\mathrm{nm}$ hasta los $860 \mathrm{~nm}$ del espectro electromagnético (Abrams y Hook, 2002). Las bandas S4 a S9 corresponden al infrarrojo de onda corta o "Short Wave InfraRed" (SWIR), tienen una resolución espacial de 30 metros y desde el año 2008 presentan anomalías que hacen los datos inutilizables (Tan, 2004). Las bandas T10 a T14 corresponden a información térmica y son las bandas "Thermal InfraRed" (TIR), tienen una resolución de 90 metros y cuentan con 4096 niveles digitales (12 bits) por banda.

Dadas las características de las imágenes Aster solamente es posible aplicar la corrección atmosférica a las bandas V1, V2 y V3N, los datos de Aster ya cuentan con corrección radiométrica, sin embargo han sido redimensionados para facilitar su compresión. La fórmula para descomprimir los datos, tal como lo especifican Abrams y Hook (2002) en la ecuación 2.

$$
\text { Radiancia }(L)=(N D-1) * U C C(\text { Ecuación } 2)
$$

Donde UCC (Unit Conversion Coefficients) corresponde al coeficiente de unidades de conversión que es distinto para cada banda y según el tipo de ganancia con la cuál fue capturada la imagen (la información de ganancia por banda está disponible en los metadatos de la imagen y puede ser ganancia alta, normal o baja). La tabla 1 corresponde a las UCC de cada banda según el tipo de ganancia.

Tabla 1. Coeficientes de conversión de unidades (UCC, por sus siglas en inglés) para las bandas V1, V2, V3N y V3B del Sensor Aster.

\begin{tabular}{|c|c|c|c|}
\hline Banda & \multicolumn{3}{|c|}{ UCC $\left(\mathbf{W} /\left(\mathbf{m}^{2 *} \mathbf{s} \mathbf{r}^{*} \boldsymbol{\mu m}\right)\right)$} \\
\hline V1 & Ganancia Alta & Ganancia Normal & Ganancia Baja \\
\hline V2 & 0,676 & 1,688 & 2,25 \\
\hline V3N/V3B & 0,708 & 1,415 & 1,89 \\
\hline
\end{tabular}

Fuente: USGS, 2014.

La aplicación de estos valores se hace por medio de la herramienta Band Math, sustituyendo el valor de ND por la banda a la cual se le va a aplicar el coeficiente. Por ejemplo, para la banda 1 la fórmula a utilizar en 
Heileen Aguilar Arias, Rodolfo Mora Zamora, Christian Vargas Bolaños. Metodología para la corrección atmosférica de imágenes Aster, RapidEye, Spot 2 y Landsat 8 con el módulo FLAASH del software ENVI

ENVI sería 1.688*(b1-1) (para que ENVI reconozca el separador de decimales, debe ser un punto). Una vez aplicados los valores de conversión la imagen resultante estará representada en unidades de radiancia.

Las imágenes de tipo AST14OTH presentan cada banda en un archivo de tipo TIFF distinto. Para aplicar la corrección atmosférica es necesario realizar una composición de bandas y asegurarse que los datos estén representados en formato BIL, de manera que se obtenga una sola imagen de tres bandas correspondientes a V1, V2 y V3N, en ese orden. Posteriormente, se deben asignar a cada banda los valores medios de longitud de onda y FWHM de la tabla 2, calculada a partir de los valores del rango espectral descritos por Abrams y Hook (2002, p.10).

Tabla 2: Longitud de onda y FWHM en $\mu \mathrm{m}$ de las bandas V1 V2 y V3 del Sensor Aster

\begin{tabular}{|c|c|c|c|c|}
\hline Banda & Mínimo & Máximo & Medio & $\begin{array}{c}\text { FWHM } \\
\text { (max-min) }\end{array}$ \\
\hline V1 & 0,520 & 0,600 & 0,560 & 0,080 \\
\hline V2 & 0,630 & 0,690 & 0,660 & 0,060 \\
\hline V3 & 0,780 & 0,860 & 0,820 & 0,080 \\
\hline
\end{tabular}

Fuente: Abrams y Hook (2002, p.10)

Para ingresar estos datos a la imagen se debe editar el encabezado de cada una de las bandas, de lo contrario no será posible realizar la corrección con FLAASH a partir del formato de datos empleados. Posterior a ello, lo que resta es hacer una composición de las bandas del visible para que se ejecute la corrección sobre la imagen completa.

Terminada la preparación de los datos, se puede aplicar la corrección atmosférica de la imagen VNIR de Aster. Dado que las bandas VNIR son tomadas por un sensor ortogonal a la superficie de la tierra, no es necesario introducir la información de inclinación del sensor (FLAASH asume que el sensor es ortogonal si no se detalla el ángulo de inclinación) y por las características de las bandas VNIR de Aster, no es posible aplicar los procedimientos de extracción de agua y extracción de aerosoles, por lo que es necesario, para estas imágenes, seleccionar un modelo atmosférico y un modelo de aerosoles según las condiciones de captura de la imagen (estos parámetros ya se han descrito en el modelo general de corrección atmosférica). 
El factor de escala para este caso corresponde a 10, mientras que el resto de valores que solicita FLAASH se ingresan de acuerdo a las características de cada imagen (fecha y hora de toma de datos). Una vez realizada la corrección atmosférica se tendrá una imagen compuesta en niveles de reflectancia lista para utilizar en otros estudios.

\section{Corrección atmosférica Spot 2}

El Satélite Para la Observación de la Tierra (SPOT) por sus siglas en francés, es una familia de sensores espaciales que utilizan la teledetección para realizar la toma de información del suelo terrestre. Este programa de sensores fue iniciado en la década de los setentas por el Centro Nacional de Estudios Espaciales (CNES) de Francia, en colaboración con Bélgica y Suecia. Desde entonces, el programa Spot ha construido cuatro generaciones de satélites a saber: Spot 1 (1986-1990), Spot 2 (1990-2009) y Spot 3 (1993-1997) constituyen la primera generación; Spot 4 (1998-2013) la segunda generación; Spot 5 (2002) la tercera generación y la cuarta generación la conforman el Spot 6 (2012) y Spot 7 que se prevé sea lanzado en 2014 (Apollo Mapping, 2013).

Las imágenes que se generan a partir de los satélites Spot se distribuyen generalmente a través de un formato de distribución de dominio público llamado DIMAP, lo que facilita el acceso a la información técnica; como por ejemplo el metadato, la descripción del producto, la georeferenciación, las condiciones y uso. En este artículo se muestra el procedimiento para corregir atmosféricamente imágenes del sensor Spot 2, nivel $1 \mathrm{~B}$, que contiene corrección radiométrica y geométrica de las distorsiones internas de la imagen.

La imagen se debe abrir en el software ENVI 32 bits con el uso del archivo dimap o dim desde File/Open External File/SPOT/dimap. El siguiente paso es realizar la conversión de niveles digitales a valores de radiancia para cada una de las bandas, para ello se hace uso de la ecuación 3 reportada por CNES (2007, p. 2).

$$
\text { Radiancia }\left(L_{k}\right)=X_{k} /\left(A_{k}^{*} G_{m k}\right)(\text { Ecuación } 3)
$$


Heileen Aguilar Arias, Rodolfo Mora Zamora, Christian Vargas Bolaños. Metodología para la corrección atmosférica de imágenes Aster, RapidEye, Spot 2 y Landsat 8 con el módulo FLAASH del software ENVI

Donde:

$k=$ corresponde a la banda espectral.

$L_{k}=$ Radiancia medida por el sensor en el techo de la atmósfera (TOA) (Watts/ $\left.\left(\mathrm{m}^{2 *} \mathrm{srad}^{*} \mu \mathrm{m}\right)\right)$ para la banda $\mathrm{k}$.

$X_{k}=$ Números Digitales para la banda $k$ (para el caso de ENVI se coloca una letra que representa la banda según la cual se está trabajando). $A_{k}=$ coeficiente de calibración absoluta para la banda k (disponibles en los metadatos de cada imagen).

$G_{m k}=$ valor de ganancia electrónica donde $\mathrm{G}_{\mathrm{mk}}=1,3^{\mathrm{m}-3}$, $\mathrm{mk} \in[1,8]$. El valor de $\mathrm{m}$ está disponible en los metadatos para cada imagen.

Con el uso de la herramienta Band Math de ENVI se ingresa una a una las fórmulas para cada una de las bandas. Una vez aplicada la fórmula se obtiene para cada una de las bandas en la imagen la conversión de los valores digitales con los que viene por defecto la imagen a valores de radiancia. El siguiente paso es guardar cada una de las bandas con su respectivo nombre y modificar la información del encabezado con respecto a los valores del punto medio de longitud de onda para cada banda (colocar unidades de micrómetros), así como los FWHM con base a los datos proporcionados en la tabla 3.

Tabla 3. Valores de longitud de onda y FWHM en $\mu \mathrm{m}$ para las bandas XS1, XS2 y XS3 del sensor Spot 2

\begin{tabular}{|c|c|c|c|c|}
\hline Banda & Mínimo & Máximo & Punto Medio & $\begin{array}{c}\text { FWHM } \\
\text { (max-min) }\end{array}$ \\
\hline B1 Green (XS1) & 0,500 & 0,590 & 0,545 & 0,09 \\
\hline B2 Red (XS2) & 0,610 & 0,680 & 0,645 & 0,070 \\
\hline B3 Near Infrared (XS3) & 0,780 & 0,890 & 0,835 & 0,110 \\
\hline
\end{tabular}

Fuente: Spot Image (2010, p.3).

Posteriormente se deben unificar las bandas en una sola imagen, para ello se utiliza la herramienta Layer Stack lo cual da como resultado una imagen compuesta por las tres bandas de color RGB, y en un formato de intercalamiento BSQ. Lo siguiente es la conversión de la imagen de un intercalamiento de BSQ a BIL, además asegurarse que la imagen se encuentre en un tipo de dato punto flotante (floating point). 
Una vez que los datos se encuentran en formato de punto flotante se asigna 1 como factor de escala para todas las bandas (ITT, 2009, p.18). Finalmente se realiza la entrada de los parámetros como por ejemplo: nombre de salida de la imagen, nombre de salida de los archivos de FLAASH, la altura del sensor (en el caso de Spot 2 de $832 \mathrm{~km}$ ) y los datos relacionados con la captura de la imagen (fecha y hora, centro de la imagen).

El sensor Spot 2 tiene una inclinación con respecto a la superficie de la tierra de $98,7^{\circ}$ (off-nadir) (CRISP, 2001), por lo cual debe corregirse el ángulo de inclinación de la captura de la imagen. En la sección de configuración avanzada del módulo FLAASH se pueden ajustar los datos del ángulo de visión del sensor al momento de la captura. Se deben ajustar dos parámetros: Zenith (corresponde al ángulo presente entre la línea de visión del satélite y el cenit de la tierra en el punto medio de la escena) y el $A z i$ muth (corresponde a la orientación del satélite con respecto al norte geográfico visto desde la tierra) (ITT, 2009). En los metadatos de una imagen Spot 2 se encuentra el valor del ángulo de incidencia (INCIDENCE_ANGLE), el cual indica el ángulo entre la línea de visión desde el satélite y el cenit de la superficie de la tierra visto desde el punto central de la imagen (Janée, 2007). Dado que FLAASH requiere el ángulo pero visto desde el satélite es necesario corregir este valor de la siguiente forma:

$$
Z=180-|\beta|(\text { Ecuación 4) }
$$

Donde:

\section{$Z=$ Parámetro Zenith de FLAASH}

$\beta=$ Valor del ángulo de incidencia obtenido de los metadatos de Spot.

El parámetro Azimuth se obtiene a partir del valor del ángulo de orientación de la escena (SCENE_ORIENTATION) presente en los metadatos de la imagen:

$$
A=-\sigma(\text { Ecuación 5) }
$$


Heileen Aguilar Arias, Rodolfo Mora Zamora, Christian Vargas Bolaños. Metodología para la corrección atmosférica de imágenes Aster, RapidEye, Spot 2 y Landsat 8 con el módulo FLAASH del software ENVI

Donde:

$A=$ Parámetro Azimuth de FLAASH

$\sigma=$ Ángulo de orientación de la escena obtenido de los metadatos de Spot.

Al finalizar con la inclusión de todos los parámetros se obtiene una imagen Spot corregida en valores de reflectancia.

\section{Corrección atmosférica para datos Landsat 8-OLI-TIRS}

El programa Landsat es una serie de satélites construidos y desarrollados por la Administración Nacional de la Aeronáutica y del Espacio (NASA, por sus siglas en inglés) en conjunto con el Servicio Geológico de los Estados Unidos (USGS, por sus siglas en inglés) (USGS, 2013).

Durante estas cuatro décadas el programa Landsat ha desplegado ocho satélites, el primero lanzado en el año de 1972 y el último, y más reciente lanzado en 2013 (Landsat 8). Para este caso se muestra la corrección atmosférica de imágenes Landsat 8 nivel 1T en formato GeoTIFF, con 30 metros de resolución espacial (multiespectral) y sistema de proyección UTM, Datum WGS84. La toma de estas imágenes inició en 2013 por parte del programa Landsat, con instrumentos de barrido óptico "Operational Land Imager" (OLI) y un sensor térmico infrarrojo "Thermal Infrared Sensor" (TIRS).

Dado que el sensor Landsat 8 es tan reciente, las versiones anteriores de ENVI no tienen la funcionalidad adecuada para procesar correctamente estos datos, por lo que es necesario utilizar la versión ENVI 5.0 Service Pack 3 con la actualización para archivos $O L I$ de Landsat 8 (envi50sp3_r4). Para abrir la imagen se accede al archivo que contiene la extensión_MTl.txt, que corresponde al formato de imagen con metadatos disponible para Landsat 8, este se abre desde la opción de File/Open as/Landsat/GeoTIFF with Metadata. El primer paso para la corrección es la conversión a radiancia. Para esto se debe ir a la opción de Toolbox/Radiometric Correction/Radiometric Calibration; la imagen inicialmente se despliega dividida en 5 segmentos: multiespectral (que posee los infrarrojos), la banda cirrus, térmica, pancromática y calidad. De todas las opciones que se muestran se debe seleccionar la que termina con MultiSpectral y asignar los diferentes valores de calibración radiométrica según se especifica en la tabla 4. 
Tabla 4. Parámetros de calibración radiométrica Landsat 8

\begin{tabular}{|l|l|}
\hline \multicolumn{1}{|c|}{ Campo } & \multicolumn{1}{c|}{ Valor } \\
\hline Tipo de calibración & Radiancia \\
\hline Intervalo de salida & BIL \\
\hline Tipo de dato de salida & Flotante \\
\hline Factor de escala & 0.10 \\
\hline Directorio de salida & Nombre y directorio de salida \\
\hline
\end{tabular}

Fuente: elaboración propia con base en: ITT (2009)

Al finalizar se obtiene una imagen con el cambio de valores de Números Digitales a valores de radiancia, además el tipo de formato de imagen en el archivo de salida es .dat. Esta imagen de datos conserva la información que viene del metadato en formato BIL.

Como paso siguiente, se procede a realizar el proceso de la corrección atmosférica. Para ello se abre el archivo de salida (el .dat), posteriormente ir a la herramienta de FLAASH y en el factor de escala utilizar para la calibración es 1 . Automáticamente se deben leer del archivo de la imagen los valores de latitud (lat) y longitud (long). Respecto de los parámetros requeridos, en el tipo de sensor dejar la opción desconocidomsi (UNKNOWN-MSI), en altitud del sensor se debe poner $705 \mathrm{~km}$ y el tamaño de pixel se coloca automáticamente, al igual que los valores de lat-long. La elevación variará en cada una de las imágenes que se vayan a calibrar. Además la fecha del vuelo y el tiempo de vuelo se deben extraer del metadato.

Las bandas de color rojo (654.6nm) e Infrarojo de onda corta 2 $(2201 \mathrm{~nm})$ de las imágenes de Landsat 8 pueden ser utilizadas para extracción de aerosoles por medio del método Kaufman-Tanre, de acuerdo a ITT (2009) este método sirve para estimar de forma más precisa la visibilidad presente en la imagen, por medio de la iteración de valores de visibilidad utilizando razones de cambio entre los valores de píxeles oscuros entre bandas cercanas a los $660 \mathrm{~nm}$ (canal bajo) y $2200 \mathrm{~nm}$ (canal alto).

Para ello se debe seleccionar el método de extracción de aerosoles de dos bandas $(K-T)$, como paso adicional se debe ir a la configuración multiespectral y en ella buscar la opción de filtro archivo de función: y colocar la siguiente dirección: "Ruta de instalación de ENVI |Files $\mid$ Exelis $\mid$ ENVI50|classic|filt_func|ldcm_oli.sli”. La selección del modelo 
Heileen Aguilar Arias, Rodolfo Mora Zamora, Christian Vargas Bolaños. Metodología para la corrección atmosférica de imágenes Aster, RapidEye, Spot 2 y Landsat 8 con el módulo FLAASH del software ENVI

atmosférico, modelo de aerosoles y visibilidad inicial dependerá de las condiciones de la imagen; finalmente al ejecutar la corrección atmosférica la imagen resultante contiene los datos en reflectancia espectral.

\section{Conclusiones}

El uso de la herramienta FLAASH requiere diferentes pasos previos para aplicar la corrección atmosférica, en los sensores, estos pasos se aplican de forma distinta, lo cual depende de las características técnicas de cada sensor.

ENVI 5 es funcional para procesar diversos sensores satelitales disponibles actualmente en el mercado y posee suficientes herramientas para aplicar a todas las imágenes utilizadas, los pasos previos a la corrección atmosférica requeridos por FLAASH.

Los metadatos y la información técnica de la imagen son indispensables para poder aplicar la corrección atmosférica. De la misma forma la imagen a corregir debe utilizarse sin alteraciones o procesamientos previos.

El módulo FLAASH no es capaz de corregir datos de los anchos de banda utilizados para capturar imágenes térmicas.

\section{Referencias}

Abrams, M. \& Hook, S. (2002). Aster user handbook. Recuperado de: http://asterweb.jpl.nasa.gov/content/03_data/04_documents/ aster_user_guide_v2.pdf

Adler-Golden, S., Berk, A., Bernstein, L.S. \& Richtsmeier, S. (1998). FLAASH, a MODTRAN4 atmospheric correction package for hyperspectral data retrievals and simulations. Recuperado de: http:// popo.jpl.nasa.gov/pub/docs/workshops/98_docs/2.pdf

Apollo, Mapping. (2013). SPOT 1-7. Recuperado de: https://apollomapping.com/imagery/medium-resolution-satellite-imagery/spot

Berk, A., Anderson, G.P., Acharya, P.K., Chetwynd, J.H., Bernstein, L.S., Shettle, E.P., Matthew, M.W. \& Adler-Golden, S.M. (2000). MODTRAN4 user's manual. Recuperado de ftp://ftp.pmodwrc.ch/pub/ Vorlesung\%20K+S/MOD4_user_guide.pdf

BlackBridge. (2013a). Rapideye: the space segment. Recuperado de: http://blackbridge.com/rapideye/about/satellites.htm 
BlackBridge. (2013b). Imágenes Satelitales Especificaciones Técnicas. Recuperado de: http://www.blackbridge.com/rapideye/upload/RE Product_Specifications_SPA.pdf

Castillo, O.G. (2012). Aplicación espectral y topológica en el procesamiento de imágenes satelitales. (Tesis de Maestría). Universidad Nacional de Colombia, Manizales, Colombia.

Centre for Remote Imaging, Sensing \& Processing (CRISP). (2001). SPOT (Satellite Pour l'Observation de la Terre), France. Recuperado de: http://www.crisp.nus.edu.sg/ research/tutorial/spot.htm

Centre National d'Etudes Spatiales (CNES). (2007). SPOT image quality perfomances. Recuperado de http://www.spot.ucsb.edu/spot-performance.pdf

Chuvieco, E. (2008). Teledetección ambiental. La observación de la tierra desde el espacio. (3. ${ }^{\mathrm{a}}$ ed.). Barcelona: Editorial Ariel, S.A.

Edwards, A. J. (1998). Lesson 3: Radiometric correction of satellite images: when and why radiometric correction is necessary. Recuperado de http://www.ncl.ac.uk/tcmweb/bilko/module7/lesson3.pdf

EXELIS. (2014). Radiance and scale factors background. Recuperado de http://www.exelisvis.com/docs/BackgroundRadianceScaleFactors.html Hedley, J.D. (2013). Hyperspectral Applications. Por James A. Goodman, Samuel J. Purkis y Stuart R. Phinn (Eds.). Coral Reef Remote Sensing. A guide for mapping, monitoring and management. (p. 90). USA: Springer.

International Telephone and Telegraph (ITT). (2009). Atmospheric Correction Module: QUAC and FLAASH User's Guide. Recuperado de: http://www.exelisvis.com/portals/0/pdfs/envi/Flaash_Module.pdf

Janée, G. (2007). SPOT Mirror and Incidence Angles. Recuperado de: $\mathrm{http} / / /$ legacy.alexandria.ucsb.edu/ gjanee/spot/docs/angles.pdf

Kruse, F.A. (2004). Comparison of ATREM, ACORN, and FLAASH atmospheric corrections using low-altitude AVIRIS data of Boulder, CO. Recuperado de http://ww.w.hgimaging.com/PDF/Kruse-JPL2004_ ATM_Compare.pdf

Lamolda, H. (2008). Estudio de la influencia de las correcciones a imágenes Landsat ETM+ en la obtención de propiedades de cubiertas vegetales. Madrid: Universidad Politécnica de Madrid. 
Heileen Aguilar Arias, Rodolfo Mora Zamora, Christian Vargas Bolaños. Metodología para la corrección atmosférica de imágenes Aster, RapidEye, Spot 2 y Landsat 8 con el módulo FLAASH del software ENVI

López, E. (1991). Corrección atmosférica en el espectro solar. Por J. Meliá (Ed). La teledetección en el seguimiento de los fenómenos naturales. Recursos renovables: Agricultura. Valencia: Universitat de València. Rejas, J. (2008). Tratamiento digital previo de las imágenes. Recuperado de http://api.eoi.es/api_v1_dev.php/fedora/asset/eoi:45518/componente45516.pdf

Saleska, S., Didan, K., Huete, A., Da Rocha, H. (2007). Amazon forests green-up during 2005 drought. Science. 318,612.

Samanta, A., Ganguly, S., Hashimoto, H., Devadiga, S., Vermonte, E., Knyazikhin, Y., Nemani, R.R. \& Myneni R.B. (2010). Amazon forests did not green-up during the 2005 drought. Geophysical research letters. 37: (LO5401). doi: 10.1029/2009g1042154

Sobrino, P., García-Collado, A.J., Sobrino, J.A. y Boluda, R. (1997). Corrección atmosférica de imágenes Landsat-5: Aplicación al estudio edafológico en la Comarca La Plana de Requena-Utiel. Por C. Hernández y J.E Arias (Eds). Actas del VII Congreso Nacional de Teledetección: Teledetección aplicada a la gestión de recursos naturales y medio litoral marino. Universidad de Santiago de Compostela-Asociación Española de Teledetección. Santiago de Compostela (191-194).

Spot Image. (2010). Spot satellite technical data. Recuperado de http:// www2.astrium-geo.com/files/pmedia/public/r329_9_spotsatellitetechnicaldata_en_sept2010.pdf

Tagestad, J.D. (2000). Radiometric standardization of adjacent Landsat Thematic Mapper images for multi-scene mosaics. (Tesis de Maestría). Utah State University, Logan, Utah.

Tan, H. (2004). ASTER: Advanced Spaceborne Thermal Emission and Reflection Radiometer. Recuperado de http://asterweb.jpl.nasa.gov/swir-alert.asp

United States Geological Survey (USGS). (2013). Landsat Project Description. Recuperado de http://landsat7.usgs.gov/about_project descriptions.php

United States Geological Survey (USGS). (2014). On Demand Registered Radiance at the Sensor-Orthorectified. AST14OTH. Recuperado de: https://lpdaac.usgs.gov/products/aster products table/ast14oth

Weisstein, E. (2014). Full width at half maximum - A Wolfram web resource. Recuperado d: http://mathworld.wolfram.com/FullWidthatHalfMaximum.html 